\title{
A TWO-DIMENSIONAL THERMODYNAMIC MODEL TO PREDICT HEART THERMAL RESPONSE DURING OPEN CHEST PROCEDURES
}

\begin{abstract}
F. G. Dias,
J. V. C. Vargas,

and M. L. Brioschi

Universidade Federal do Paraná

Departamento de Engenharia Mecânica

Centro Politécnico

Bairro Jardim das Américas

CP. 19011, Curitiba, Paraná, Brasil

gallego@ufpr.br

ABSTRACT

In this work, the temperature distribution of the heart in an open chest surgery scenario is studied. It is also evaluated the cardiac thermal effects of the injection of a cooling liquid in the aorta root, which is used in infrared thermography. The finite element method was used to develop a model that predicts the temperature distribution modification in a 2-dimensional slice of the heart. This thermodynamic model allows the computational simulation of the thermal cardiac response to open chest procedures, which are required by cardiac surgery. The influence of several operating parameters (e.g., coronary flow rate, temperature) on the resulting thermal distribution is analyzed. Therefore, this analysis allows the identification of parameters that could be controlled to minimize the loss of energy, and consequently, avoiding the hazardous thermal distribution that could put the heart in danger during cardiac surgery.
\end{abstract}

Received: April 06, 2016

Revised: May 16, 2016

Accepted: June 16, 2016

Keywords: heart thermodynamic model, heart thermal distribution, heart thermography, finite element heart model, cardiac surgery

\section{NOMENCLATURE}

$\mathrm{c}_{\mathrm{p}}$ fluid specific heat at constant pressure, $\mathrm{J} /(\mathrm{kg} . \mathrm{K})$

K fluid thermal conductivity, W/(m.K)

m total mass, kg

$\tilde{\mathrm{m}}$ dimensionless total mass

p pressure, $\mathrm{N} / \mathrm{m}^{2}$

$\mathrm{P} \quad$ dimensionless pressure

Pr fluid Prandtl number, $v / \alpha$

$\tilde{\mathrm{q}}$. dimensionless overall thermal conductance,

Q overall heat transfer rate, $\mathrm{W}$

Re Reynolds number, $\mathrm{u}_{\infty} 2 \mathrm{~b} / \mathrm{v}$

$\mathrm{T}$ average fluid temperature, $\mathrm{K}$

$\mathrm{u}_{1}, \mathrm{u}_{2}, \mathrm{u}_{3}$ velocity components, $\mathrm{m} / \mathrm{s}$

$\mathrm{U}_{1}, \mathrm{U}_{2}, \mathrm{U}_{3}$ dimensionless velocity components

$\mathrm{x}, \mathrm{y}, \mathrm{z}$ cartesian coordinates, $\mathrm{m}$

$\mathrm{X}, \mathrm{Y}, \mathrm{Z}$ dimensionless cartesian coordinates

\section{Greek symbols}

$\alpha \quad$ thermal diffusivity, $\mathrm{m}^{2} / \mathrm{s}$

$\gamma \quad$ Penalty factor

$\theta \quad$ dimensionless temperature

$v \quad$ fluid kinematic viscosity, $\mathrm{m}^{2} / \mathrm{s}$

$\rho \quad$ density, $\mathrm{kg} / \mathrm{m}^{3}$

\section{INTRODUCTION}

Brazil has one the highest rates of death by heart disease in the world (34\%). One third of those cases are caused by coronary artery disease which is the main reason of sudden death, infarction, angina and cardio pathology, according to the Brazilian Society of Cardiology (2000).

In cases of coronary occlusions, the most common solution is the revascularization procedure by using either arterial or venous grafts. An inadequate or insufficient anastomosis results in another surgery or even leads the patient to death by infarction either during or after surgery.

As surgery procedure is highly recommended in these cases, it inevitably exposes the mediastinal space, a closed space never meant to have direct contact with the exterior environment, directly to the environment's hazardous agents, such as viruses, bacterial colonies, and the extremely low temperature of the operating room.

The present work focus the thermal distribution of the heart in an open chest surgery scenario, and, as most of open chest surgeries concerns revascularization, a feasibility correlation with the left coronary flow is conducted based on the change of the temperature along the pericardia tissue surrounding the left anterior descending (LAD) branch of the left coronary artery.

The approach consists on a scale analysis of the mass, energy and momentum balance equations of the myocardium, pericardium tissue and of the blood flow using finite elements, letting the user to change the main parameters of the mesh, thus calculating the new equilibrium for the altered simulating environment.

To ensure the convergence of the steady-state solution and the proper variation of the physiological and environmental parameters, appropriate nondimensional groups are defined and the results 
reported in dimensionless charts.

\section{THEORY}

Figure 1 shows a 3D model of the heart. This model is very precise and will help the reader to understand what the present study is engaged to accomplish. Note that this is the anterior view of the heart in cardioplegic status, with the patient in supine position, and with his thoracic cavity opened.

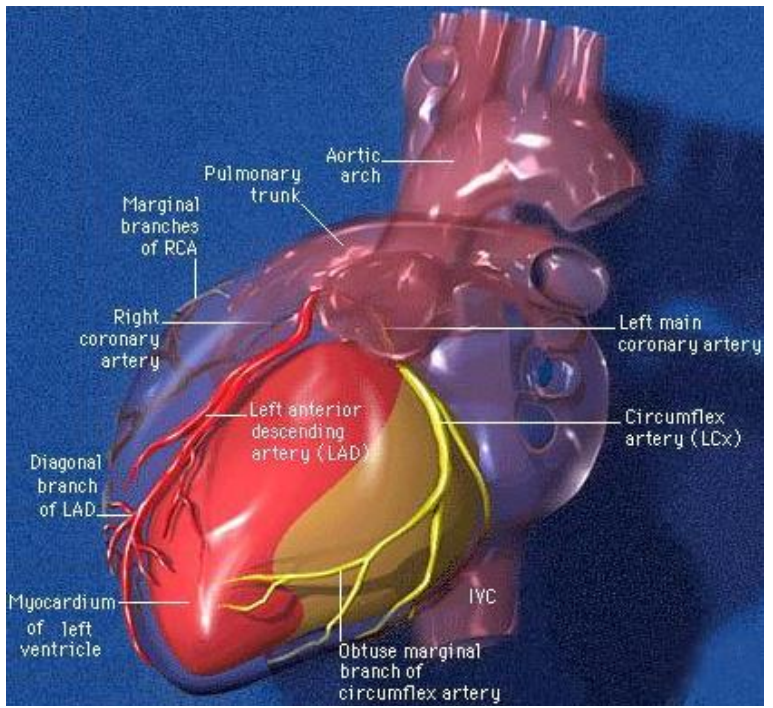

Figure 1. 3D model of the heart [Yale medicine website - http://www.info.med.yale.edu].

Figure 2 shows, using radioactive marked radiograph, the chosen $2 \mathrm{D}$ slice of the heart and on its right is shown with the yellow line where the slice is located in the heart. As the model is built using a 2D slice of the heart, it is crucial for the precise modeling and simulation that this slice is representative of the hole 3D heart, so in every slice in the same axial line, there must exist the same structures represented, resulting derivates of velocities and temperatures between the adjacent slices to be zero.

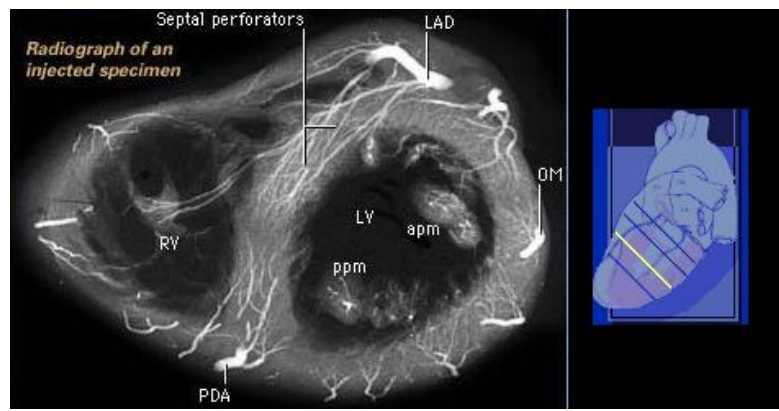

Figure 2. Axial slice of the heart to be modeled and its location in the real heart [Yale medicine website http://www.info.med.yale.edu].

Figure 3 shows the general sketch of the problem configuration of Fig. 2. As the main objective of this article is the modeling of the heart thermal response during open chest surgery, the heart is supposedly half immersed into the pericardia domain, represented by the (A) portion. The (B) portion represents the area of the heart that is exposed into the operating room hazardous environment. Portion (C) represents the blood flowing into the ventricles and into the capillaries from the left anterior descending branch of the left coronary to the vein tributary of the coronary sinus. Portion (D) represent the myocardium itself and Portion (E) represents the blood that has a known flow out from the left anterior descending artery branch.

The duct that is connecting the left anterior descending branch and the vein tributary of the coronary sinus corresponds to the array of capillaries of the plane of the slice analyzed, so, in the adjacent slices is found the same similar structures. Thus, as noticed before, the derivate of the velocities and temperatures from one slice into other is zero, which allows the bidimensional modeling of the given tridimensional problem by the analysis of only one slice, once the same picture repeats approximately in every adjacent slices (or planes).

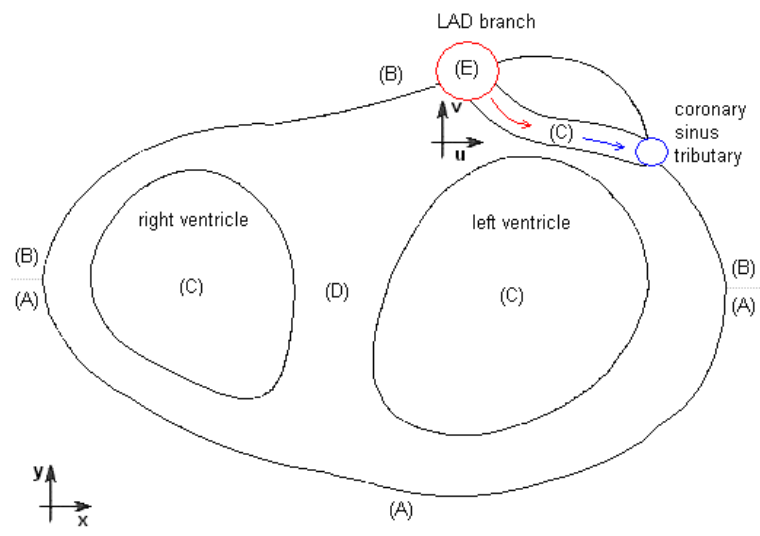

Figure 3. Problem sketch and computational domain.

In the present work, blood is considered a Newtonian fluid. Perhaps the earliest attempts to study the flow properties of blood were made by Young and Poiseuille in the 17th century and simulating blood flow in complex corporeal and clinical environment is still a difficult problem. The complexities of simulating blood flow were realized quite early (Fox and Saibel, 1963). It is well established now that blood is a non-Newtonian fluid. However, most analysis are performed considering blood as a Newtonian fluid which works well in large and medium arteries with simple geometries, as is the case of the left anterior descendent branch of the left coronary and the ventricles. For most other interesting and challenging problems the nonNewtonian effects have to be considered.

$$
\frac{\partial u}{\partial x}+\frac{\partial v}{\partial y}=0
$$




$$
\begin{gathered}
u \frac{\partial u}{\partial x}+v \frac{\partial u}{\partial y}=-\frac{1}{\rho} \frac{\partial p}{\partial x}+v\left(\frac{\partial^{2} u}{\partial x^{2}}+\frac{\partial^{2} u}{\partial y^{2}}\right) \\
u \frac{\partial v}{\partial x}+v \frac{\partial v}{\partial y}=-\frac{1}{\rho} \frac{\partial p}{\partial y}+v\left(\frac{\partial^{2} v}{\partial x^{2}}+\frac{\partial^{2} v}{\partial y^{2}}\right)+g \beta T \\
u \frac{\partial T}{\partial x}+v \frac{\partial T}{\partial y}=\alpha\left(\frac{\partial^{2} T}{\partial x^{2}}+\frac{\partial^{2} T}{\partial y^{2}}\right)
\end{gathered}
$$

In dimensionless form, Eqs. (1)-(4) can be written as:

$$
\frac{\partial \mathrm{U}}{\partial \mathrm{X}}+\frac{\partial \mathrm{V}}{\partial \mathrm{Y}}=0
$$

$$
\begin{aligned}
\frac{\mathrm{Ra}^{1 / 2}}{\operatorname{Pr}}\left[\mathrm{U} \frac{\partial \mathrm{U}}{\partial \mathrm{X}}+\mathrm{V} \frac{\partial \mathrm{U}}{\partial \mathrm{Y}}\right] \\
=-\frac{\partial \mathrm{P}}{\partial \mathrm{X}}+\frac{\partial^{2} \mathrm{U}}{\partial \mathrm{X}^{2}}+\frac{\partial^{2} \mathrm{U}}{\partial \mathrm{Y}^{2}}
\end{aligned}
$$

$$
\begin{gathered}
\frac{\mathrm{Ra}^{1 / 2}}{\operatorname{Pr}}\left[\mathrm{U} \frac{\partial \mathrm{V}}{\partial \mathrm{X}}+\mathrm{V} \frac{\partial \mathrm{V}}{\partial \mathrm{Y}}\right] \\
=-\frac{\partial \mathrm{P}}{\partial \mathrm{Y}}+\frac{\partial^{2} \mathrm{~V}}{\partial \mathrm{X}^{2}}+\frac{\partial^{2} \mathrm{~V}}{\partial \mathrm{Y}^{2}}+\mathrm{Ra}^{1 / 2} \theta \\
\mathrm{Ra}^{1 / 2}\left[\mathrm{U} \frac{\partial \theta}{\partial \mathrm{X}}+\mathrm{V} \frac{\partial \theta}{\partial \mathrm{Y}}\right]=\frac{\partial^{2} \theta}{\partial \mathrm{X}^{2}}+\frac{\partial^{2} \theta}{\partial \mathrm{Y}^{2}}
\end{gathered}
$$

Where dimensionless variables have been defined as follows:

$$
\begin{gathered}
(\mathrm{X}, \mathrm{Y})=\frac{(\mathrm{x}, \mathrm{y})}{\mathrm{L}}, \quad \mathrm{P}=\frac{\mathrm{p}}{\rho \mathrm{U}_{\infty}^{2}}, \quad(\mathrm{U}, \mathrm{V})=(\mathrm{u}, \mathrm{v}) \frac{\mathrm{L}}{\alpha} \mathrm{Ra}^{-1 / 2}, \\
\theta=\frac{\mathrm{T}-\mathrm{T}_{\infty}}{\mathrm{T}_{\mathrm{w}}-\mathrm{T}_{\infty}}, \quad \mathrm{Ra}=\frac{\mathrm{g} \beta\left(\mathrm{T}_{\mathrm{w}}-\mathrm{T}_{\infty}\right) \mathrm{L}^{3}}{\alpha \nu}, \quad \operatorname{Pr}=\frac{v}{\alpha}
\end{gathered}
$$

where $(\mathrm{x}, \mathrm{y})$ are the Cartesian coordinates, $\mathrm{m}$; $\mathrm{p}$ the pressure, $\mathrm{N} / \mathrm{m}^{2}$; $\rho$ the fluid density, $\mathrm{Kg} / \mathrm{m}^{3}$; g the gravity acceleration, $\mathrm{m} / \mathrm{s}^{2}$; $\mathrm{T}$ the heart temperature, $\mathrm{K} ; \mathrm{T}_{\infty}$ the operation room temperature, $\mathrm{K} ; \mathrm{T}_{\mathrm{w}}$ the ventricular blood temperature, $\mathrm{K} ; \beta$ the coefficient of thermal expansion, $\mathrm{K}^{-1}$; $\mathrm{L}$ the heart slice length, $\mathrm{m}$; $v$ the blood kinematics' viscosity, $\mathrm{m}^{2} / \mathrm{s}$ and $\alpha$ is the blood thermal diffusivity, $\mathrm{m}^{2} / \mathrm{s}$; $\mathrm{cp}$ is the specific heat capacity, $\mathrm{J} / \mathrm{kg} . \mathrm{K} ; \mu$ is the dynamic viscosity, Pa.s; $\mathrm{k}$ is the thermal conductivity, $\mathrm{W} / \mathrm{m} . \mathrm{K}$.

To complete the problem formulation, the following boundary conditions are specified for the extended computational domain of the slice model of Fig. 3, where the temperature of the operation room was set to $25^{\circ} \mathrm{C}(\theta=0)$, inner chest pericardium temperature to $35.5{ }^{\circ} \mathrm{C} \quad(\theta=0.875)$, blood temperature to $37^{\circ} \mathrm{C}(\theta=1)$ and the flow in the Left Anterior Descending branch of the Left Coronary was set to $8.8 \mathrm{~cm} / \mathrm{s},\left(\mathrm{U}=867.10^{-5}\right.$ and $\left.\mathrm{V}=5.10^{-3}\right)$ :

$$
\begin{aligned}
& \text { (A) } \mathrm{U}=0, \mathrm{~V}=0, \theta=0.875 \text {, } \\
& \text { (B) } \mathrm{U}=0, \mathrm{~V}=0, \theta=0,
\end{aligned}
$$

$$
\text { (C) } \theta=1.0
$$

(D) $\mathrm{U}=0, \mathrm{~V}=0$,

(E) $\mathrm{U}=867.10^{-5}, \mathrm{~V}=5.10^{-3}, \theta=1.0$,

Once the geometry of the computational domain defined by the slice of Fig. 3 is specified, Eqs. (5)(15) deliver the resulting velocities, pressure and temperature fields in the domain.

\section{NUMERICAL METHOD}

The numerical solution of Eqs (5)-(15) was obtained utilizing the finite element method (Zienkiewicz and Taylor, 1989). This way, the velocities and temperature fields in the model of Fig. 3 were determined.

The first step in the numerical implementation was the elimination of the pressure variable from Eqs. (6) and (7), by using a penalty model, approximating Eq. (5) as follows (Hughes et al., 1979):

$$
\frac{\partial \mathrm{U}}{\partial \mathrm{X}}+\frac{\partial \mathrm{V}}{\partial \mathrm{Y}}=\frac{\mathrm{P}}{\gamma}
$$

where $\gamma$ is the penalty factor, which must be assumed large enough in order to satisfy mass conservation approximately.

The implementation of the finite element method for the solution of Eqs. (5)-(15) starts from obtaining the variational (weak) form of the problem. Next, the weak form is discretized, by a suitable method. Due to the characteristics of the flow inside the arrangement of Fig. 3, physically, it is seen that the solution at a specified location in the model depends preferably on what happens upstream, considering the direction of the forced flow. It is well known that the Galerkin method, which is the analogue of a central differences scheme in the finite differences method, does not capture this physical aspect of in its discretized equations. Therefore, the discretization used in this work is an 'upwind' scheme proposed by Hughes (1978), where it is possible to adequate the discrete form of the problem to the physical characteristics of the flow.

After developing the discrete form of the problem, the resulting algebraic equations are arranged in a matricial form for the steady-state 2-D 
problem as follows:

$$
\begin{gathered}
{\left[\begin{array}{cc}
\mathrm{B}_{1} & 0 \\
0 & \mathrm{~B}_{2}
\end{array}\right]\left\{\begin{array}{l}
\tilde{\mathrm{T}} \\
\tilde{\mathrm{T}}
\end{array}\right\}+\left[\begin{array}{cc}
\mathrm{C}(\tilde{\mathrm{U}}) & 0 \\
0 & \mathrm{C}(\tilde{\mathrm{U}})
\end{array}\right]\left\{\begin{array}{l}
\tilde{\mathrm{U}}_{1} \\
\tilde{\mathrm{U}}_{2}
\end{array}\right\}} \\
+\left[\begin{array}{cc}
2 \mathrm{~K}_{11}+\mathrm{K}_{22} & \mathrm{~K}_{21} \\
\mathrm{~K}_{12} & \mathrm{~K}_{11}+2 \mathrm{~K}_{22}
\end{array}\right]\left\{\begin{array}{l}
\tilde{\mathrm{U}}_{1} \\
\tilde{\mathrm{U}}_{2}
\end{array}\right\} \\
+\left[\begin{array}{cc}
\hat{\mathrm{K}}_{11} & \hat{\mathrm{K}}_{12} \\
\hat{\mathrm{K}}_{21} & \hat{\mathrm{K}}_{22}
\end{array}\right]\left\{\begin{array}{c}
\tilde{\mathrm{U}}_{1} \\
\tilde{\mathrm{U}}_{2}
\end{array}\right\}=\left\{\begin{array}{l}
\mathrm{F}_{1} \\
\mathrm{~F}_{2}
\end{array}\right\}, \\
\tilde{\mathrm{D}}(\tilde{\mathrm{U}}) \tilde{\mathrm{T}}+\tilde{\mathrm{LT}}=\mathrm{G},
\end{gathered}
$$

where $\mathrm{B}_{1}$ and $\mathrm{B}_{2}$ are the buoyancy terms; $\mathrm{C}(\tilde{\mathrm{U}})$ is the capacity matrix that contains the advective terms of the momentum equations, which depends nonlinearly on the solution $\tilde{\mathrm{U}}$ (bipartitioned vector: $\tilde{\mathrm{U}}_{1}$ direction $\mathrm{X}$ and $\tilde{\mathrm{U}}_{2}$ - direction $\mathrm{Y}$, each one with a number of components equal to the number of unknowns in the mesh); $\hat{\mathrm{K}}_{11}, \hat{\mathrm{K}}_{12}, \hat{\mathrm{K}}_{21}$ and $\hat{\mathrm{K}}_{22}$ are the penalty of the pressure variable from the momentum equations by using Eq. (11), which were computed with reduced integration (one point in each direction with linear shape functions) to avoid locking; $\tilde{\mathrm{D}}(\tilde{\mathrm{U}})$ is the capacity matrix that contain the advective terms of the energy equation; $\tilde{L}$ is the diffusive matrix with constant coefficients (constant themal conductivity), that contain the diffusive terms from the energy equation; $F_{1}$ and $F_{2}$ are the force vectors of the momentum equations that contain the field forces and the velocity boundary conditions, and $\mathrm{G}$ is the heat source terms of the energy equation and the temperature boundary conditions.

For the sake of brevity, the mathematical details of the components of the above-described matrices are not presented. However, the reader is directed to the work of Reddy and Gartling (1994), which was the basis for the present study. A Fortran subroutine was written to implement the Navier-Stokes and Energy equations in two dimensions as an isoparametric, four-noded, linear element, which was the aggregated to the open code called 'finite element analysis program', FEAP, originally written by Zienkiewicz and Taylor (1989).

\section{RESULTS AND DISCUSSION}

For the present study, the mesh model was divided in three different materials, each one with the following properties:

Material 1 - Myocardium:

$$
\rho=1125 \frac{\mathrm{Kg}}{\mathrm{m}^{3}}, \mathrm{cp}=3475 \frac{\mathrm{J}}{\mathrm{Kg} \cdot \mathrm{K}}, \mathrm{k}=0.493 \frac{\mathrm{W}}{\mathrm{m} \cdot \mathrm{K}}
$$

Material 2 - Pericardium:

$$
\rho=900 \frac{\mathrm{Kg}}{\mathrm{m}^{3}}, \mathrm{cp}=2650 \frac{\mathrm{J}}{\mathrm{Kg} \cdot \mathrm{K}}, \mathrm{k}=0.201 \frac{\mathrm{W}}{\mathrm{m} \cdot \mathrm{K}}
$$

Material 3 - Blood:

$$
\begin{aligned}
& \mu=27.10^{-4} \mathrm{~Pa} . \mathrm{s}, \rho=1060 \frac{\mathrm{Kg}}{\mathrm{m}^{3}}, \\
& \mathrm{cp}=3889 \frac{\mathrm{J}}{\mathrm{Kg} \cdot \mathrm{K}}, \mathrm{k}=505.10^{-3} \frac{\mathrm{W}}{\mathrm{m} \cdot \mathrm{K}}, \\
& \beta=21.10^{-5} \frac{1}{\mathrm{~K}}, \mathrm{~g}=-9.87 \frac{\mathrm{m}}{\mathrm{s}^{2}}
\end{aligned}
$$

Initially, it was analyzed the heart thermal distribution at usual conditions for operating room , i.e., temperature of the operation room was set to $25^{\circ} \mathrm{C}(\theta=0)$, inner chest pericardium temperature to $35.5^{\circ} \mathrm{C}(\theta=0.875)$ and blood temperature to $37^{\circ} \mathrm{C}$ $(\theta=1)$.

The flow in the Left Anterior Descending branch of the Left Coronary was set to $\mathrm{U}=867.10^{-5}$ and $\mathrm{V}=5.10^{-3}$

Figure 4 shows the obtained results of the normalized temperature distribution over the heart slice.

Figure 5 shows the obtained vectors representing the results for the internal flows in the capillary duct linking the arterial feeding source to the venous draining system and the flows internal to the ventricular cavities, as this last one is only due to the temperature gradient inside the heart. Notice that the center of the vortex of each ventricle's flow vectors is located near the region of most intense temperature gradient, i.e., the transition area between the heart immersed into the pericardia domain and the heart exposed to the environment. As a matter of fact, this intense flow near the higher gradient area is explained by the buoyancy term in the momentum Eq. (2), g $\beta \mathrm{T}$, which directs the distribution of the velocities' derivates according to the temperature gradient.

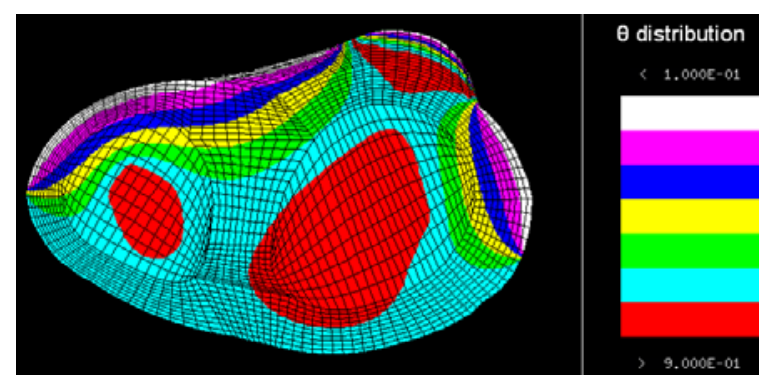

Figure 4. Cardiac thermal distribution at common conditions for operating room.

Next, it was analyzed the correlation of the LAD flow with the change of the temperature distribution along the pericardia tissue surrounding the left anterior descending (LAD) branch of the left 
coronary artery and in the myocardial tissue near the left ventricle.

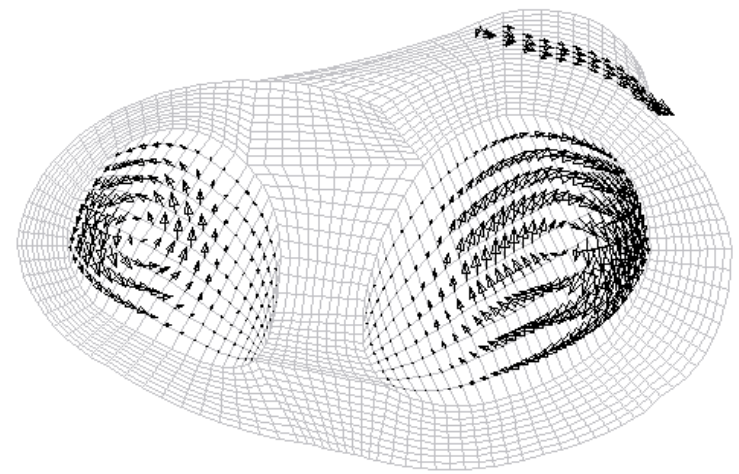

Figure 5. Internal heart blood flow vectors.

Figure 6 shows the correlation between LAD flow and the left anterior pericardial temperature, where the probed site was above the LAD and bellow the portion of the heart in direct contact with the environment, the ideal and most accessible point to get a direct measure of the temperature in revascularization or valve transplantation surgeries, e.g., using infrared imaging (Brioschi et al., 2000; Siebert et al., 2001; Brioschi et al., 2002).

Figure 7 shows the correlation between LAD flow and the myocardial tissue temperature near the left ventricle. This clearly shows that the near presence of the left ventricle is predominant and sufficient to keep this region warn, therefore reducing the dependence with the heat distribution generated by the LAD flow for this specific site.

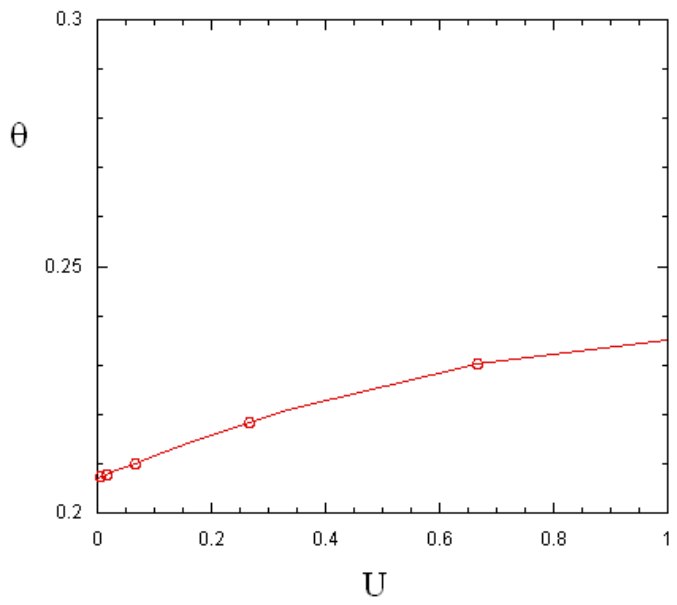

Figure 6. Correlation between LAD dimensionless flow and the left anterior pericardial dimensionless temperature.

After that, the injection of a cooling liquid at $28^{\circ}$ C $(\theta=0.25)$ in the aorta root was simulated by setting the input temperatures of the LAD branch to this chosen temperature. Figure 8 shows the heat distribution over the heart after this procedure. This maneuver is very important to estimate the LAD arterial flow using Infrared Imaging (Brioschi et al., 2002), thus allowing the medical staff to obtain a fair qualitative estimative of the flow based on the new temperature gradients trough time of the LAD surrounding tissue (pericardial fat), seconds after the liquid injection. Notice that the method is quite invasive and hazardous to the heart, so the period of injection must be shorter as possible, thus avoiding unnecessary temperature and isquemic damage to the anterior portion of the left ventricle.

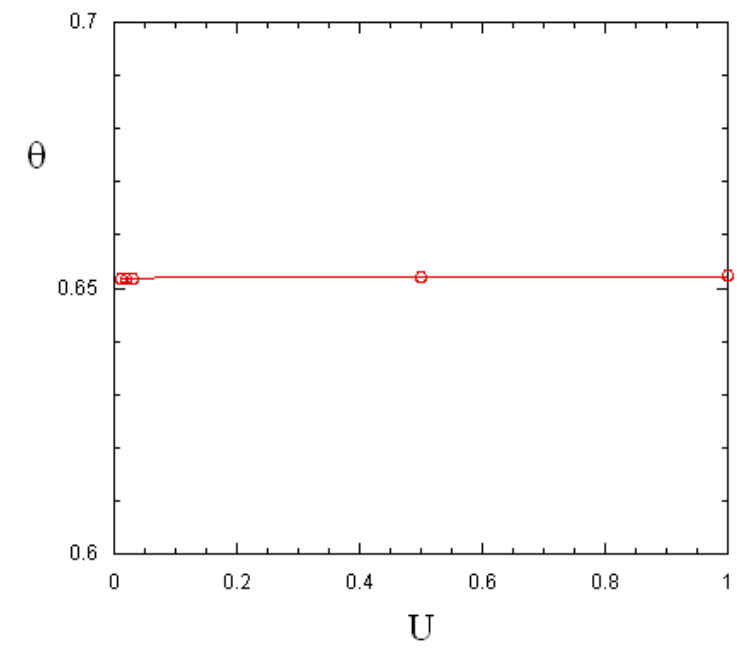

Figure 7. Correlation between LAD flow and the myocardial tissue temperature.

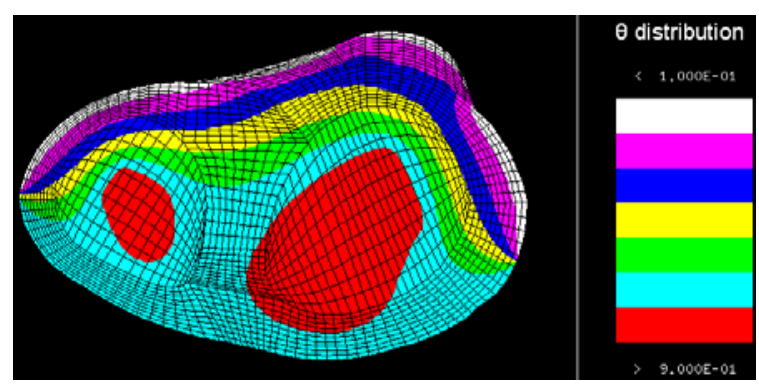

Figure 8. Thermal distribution in the injection of a cooling liquid at $28^{\circ} \mathrm{C}$ in the aorta root.

\section{CONCLUSIONS}

This study demonstrates that an open chest procedure greatly changes the thermal distribution of the heart. It provided a model that enables the physician to evaluate the temperature distribution of each desirable portion of the heart, thus providing a handful tool to predict possible temperature damages during the exposed heart surgeries.

It also provided a feasible correlation of the left coronary flow based on the change of the temperature along the pericardia tissue surrounding the left anterior descending (LAD) branch of the left coronary artery. This correlation is very important, especially upon the fact that the practical correlations 
used nowadays cross pointing LAD flow versus pericardium temperature, e.g., infrared imaging, are just qualitative, providing the surgeon a fair idea of the flow status. Nonetheless, this works shows that it's possible to obtain a quantitative calculation of the LAD flow based on the temperature of the pericardial region surrounding the left anterior descending branch, which can be accomplished in future researches.

Ultimately, computational systems that combine two-dimensional modeling with practical acquired thermal distribution parameters will enable physicians to design therapeutic and visualize procedures that yield improved surgery safety and certified blood flow to patients with cardiovascular disease.

\section{REFERENCES}

Brazilian Society of Cardiology (SBC), 2000, National Preventive and Epidemilogical Program.

Brioschi, M. L., Cimbalista, M., Colman, D., Machuca, T. N., and Loures, D. R. R., 2002, TermoCoronário-Angiografia: Padronização do Método e Primeiras Aplicações Clínicas no Brasil, Revista Brasileira de Cirurgia Cardiovascular, Vol. 17, pp. 15-19. (in Portuguese)

Brioschi, M. L., Cimbalista Jr., M., Nakagawa, C. R., Colman, D., Silveira, F., Coltro, A., von Bahten, L. C., and Brofman, P. R., 2000, Thermal Coronary Angiography for Intraoperative Avaliation of Cardiac Perfusion: Preliminary Experimental Study, Arq Med PUCPR, Vol. 2, pp. 95-98.

Fox, E. A., and Saibel, E., 1963, Attempts in Mathematical Analysis of Blood Flow, Transactions of Society of Rheology Vol. VII, pp. 25-31.

Hughes, T. J. R., 1978, A Simple Scheme for Developing Upwind Finite Elements, International Journal for Numerical Methods in Engineering, Vol. 12, pp. 1359-1365.

Hughes, T. J. R., Liu, W. K., and Brooks, A., 1979, Review of Finite Element Analysis of Incompressible Viscous Flows by Penalty Function Formulation, Journal of Computational Physics, Vol. 30, pp. 1-60.

Reddy, J. N., and Gartling, D. K., 1994, The Finite Element Method in Heat Transfer and Fluid Dynamics, CRC Press, Boca Raton, FL (Chapters 45).

Siebert, J., Keita, L., Kaczmarek, M., Anisimowicz, L., Rogowski, J., and Narkiewicz, M., 2001, Application of Intraoperative Thermography in Coronary Artery Bypass Grafting without Extracorporeal Circulation - Clinical Report, Medical science Monitor, Vol. 7, pp. 766-770.

Zienkiewicz, O. C., and Taylor, R. L., 1989, The Finite Element Method, Vol. 1, McGraw-Hill, London, (Chapter 15).
Reddy, J. N., and Gartling, J. N., 1994, The Finite Element Method in Heat Transfer and Fluid Dynamics, CRC Press.

Rocha, L. A. O., Saboya, F. E. M., and Vargas, J. V. C., 1997, A Comparative Study of Elliptical and Circular Sections in One And Two-Row Tubes and Plate Fin Heat Exchangers, International Journal of Heat Fluid Flow, Vol. 18, No. 2, pp. 247-252.

Saboya, S. M., and Saboya, F. E. M., 2001, Experiments on Elliptic Sections in One and TwoRow Arrangements of Plate Fin and Tube Heat Exchangers, Experimental Thermal and Fluid Science, Vol. 24, No. 1-2, pp. 67-75.

Zienkiewicz, O. C., and Taylor, R. L., 1989, The Finite Element Method, Vol. 1, McGraw-Hill. 